\section{Sistema Nacional de Saúde britânico: trajetória de reformas, 1990-2002}

\author{
The British National Health Service: a history of \\ reforms, 1990-2002
}

\section{Sistema Nacional de Salud británico: trayectoria de reformas, 1990-2002}

Javier Amadeo

Rosemarie Andreazza 2

Ademar Arthur Chioro dos Reis 2

\section{Resumo}

Nas últimas décadas, assistimos importantes transformações nos serviços públicos que implicam mudanças no papel do Estado como agente central no processo de regulação das relações público-privado e na definição dos níveis de financiamento público. As propostas de reforma dos sistemas de saúde formam parte do processo de transformações sociais que afetaram os diferentes países. No entanto, as mudanças na articulação das diferentes dimensões são resultado tanto de processos amplos de transformação social como também de experiencias e temporalidades específicas, fundamentais para entender os resultados desse processo. O Sistema Nacional de Saúde britânico (NHS) passou nas últimas décadas pelo processo de reforma mais importante desde a sua criação em 1948. Esse processo começou com um conjunto de medidas implantadas pelo governo conservador em 1991, mas que foram continuadas pelas alterações realizadas em 1997 e em 2002. Ao analisar a reforma realizada no NHS, para além de aportar elementos que permitam compreender o debate atual proporcionado pelas iniciativas lideradas por Boris Johnson que visam a empreender nova reforma no NHS, a partir de 2021, é possível encontrar convergências com outras propostas de reforma de sistemas públicos de saúde, que se inspiraram em sua conformação no modelo britânico, como por exemplo no caso do Brasil. O objetivo do presente texto é discutir e analisar um caso de reforma no sistema de saúde que pode ser considerado como paradigmático no desenvolvimento de grandes tendências na área. O artigo busca analisar especificamente o processo de reformas realizadas, a partir da década de 1990, no NHS, e suas consequências do ponto de vista da reestruturação do sistema de saúde.

Sistemas de Saúde; Reforma dos Serviços de Saúde; Serviços de Saúde; Saúde Pública

\author{
Correspondência \\ J. Amadeo \\ Rua dos Pinheiros 447, São Paulo, SP 05422-010, Brasil. \\ javier.amadeo@unifesp.br \\ 1 Departamento de Ciências Sociais, Universidade Federal de \\ São Paulo, São Paulo, Brasil. \\ 2 Departamento de Medicina Preventiva, Escola Paulista de \\ Medicina, São Paulo, Brasil.
}




\section{Introdução}

Os sistemas de saúde, como sustenta Immergut 1, podem ser considerados os mais complexos e controversos dentre o conjunto das políticas sociais. A discussão sobre as características desses sistemas provoca grandes debates e tomadas de posição política entre os diferentes atores sociais envolvidos. Médicos, sindicatos, formuladores de políticas, usuários dos sistemas de saúde e outros grupos de interesse estão permanentemente envolvidos nas discussões e nos conflitos sobre as orientações das políticas de saúde. Para os grupos políticos, as políticas nacionais de saúde aparecem como um espaço privilegiado para colocar clara e abertamente as suas posições ideológicas e como forma de conseguir apoio social para suas forças. A definição de políticas públicas sobre financiamento e provisão de serviços na área da saúde tem papel central na definição do caráter predominantemente público ou privado de um determinado sistema. Para a autora, as políticas públicas "têm importantes consequências para a autonomia da profissão médica, para o controle e direção dos sistemas de saúde, e para a distribuição e financiamento destes" 1 (p. 2).

Os sistemas de saúde que se desenvolveram na Europa depois da Segunda Guerra Mundial podem ser vistos como parte da expansão do Estado de bem-estar social. Vários estudos, entre os quais sobressai a análise já clássica de Marshall, têm destacado a relação entre o processo de constituição do sistema de bem-estar e o processo de expansão de direitos econômicos e sociais durante o pós-guerra 1. Um dos elementos centrais para pensar a estrutura do sistema de saúde, e suas transformações, está relacionado com um conjunto de dimensões: "extensão e tipo de financiamento público; tipo de regulação (pública e privada) que tal estrutura de financiamento proporciona; papel do Estado como proprietário dos meios de produção (de serviços e de insumos) e como empregador (direto ou indireto)" 2 (p. 762).

A forma específica que cada sistema de saúde adquiriu nos diferentes países é resultado da maneira particular segundo a qual essas três dimensões se articularam ao longo do tempo como resultado de arranjos sociais e lutas políticas de caráter mais abrangentes 1,2 .

Segundo Immergut 1, é possível construir teoricamente três grandes modelos de sistemas de saúde que foram articulados na Europa do pós-guerra com base nas dimensões anteriormente descritas. Em primeiro lugar, o modelo de subsídios a fundos mutualistas, no qual o governo subsidia as organizações privadas, que são responsáveis pela provisão de serviços de saúde. Em segundo, o modelo do seguro nacional de saúde, no qual existe financiamento público para custear o seguro. Por último, o sistema nacional de saúde, com recursos públicos via impostos para o financiamento de todos os gastos. Cada um desses modelos tem características diferentes, além do financiamento, no tipo de regulação e no papel do Estado como proprietário dos serviços de saúde 1,3.

O Sistema Nacional de Saúde (National Health Service - NHS) britânico é considerado o principal exemplo desse último tipo de serviço de saúde. Esse modelo é avaliado como mais equitativo quando comparado com os modelos anteriormente descritos. A principal característica desse sistema é que o governo garante os serviços de saúde para a população de forma direta, mediante hospitais públicos e médicos também pagos com recursos públicos. A ideia fundante é que a saúde deve ser vista como um direito da população e, como consequência, os serviços de saúde devem ser subministrados pelo Estado por meio de financiamento público, com médicos e profissionais de saúde contratados como funcionários públicos e com estabelecimentos públicos de saúde, como hospitais, clínicas, prontossocorros etc. O modelo britânico parte do conceito de que a proteção social oferecida pelo sistema de saúde deve ser um direito universal, independentemente das possibilidades do indivíduo pagar. Nesse modelo, o sistema é financiado mediante impostos gerais e o governo oferece cobertura total no tratamento médico, desde remédios, pagamento de médicos e outros profissionais de saúde até estrutura física. No caso do modelo britânico existe também um processo de centralização administrativa que busca ser um mecanismo de controle de gastos 1 .

Nas últimas décadas, o NHS passou pela reforma mais importante desde a sua criação em 1948. Esse processo começou com um conjunto de medidas implantadas pelo governo conservador em 1991, mas que foram continuadas pelas alterações realizadas em 1997 e em 2002 pelo governo trabalhista. Essa reforma teve consequências fundamentais na reestruturação do sistema de saúde e na relação público-privado como um todo.

Para Klein 4, a situação do NHS deve ser analisada com base no paradoxo que representa. O processo de reforma implicou o surgimento de elementos paradoxais no interior do sistema de saúde 
e que são fundamentais para entender os resultados alcançados nas primeiras décadas do presente século. Como consequência dessas reformas, o NHS é o único sistema na Europa que durante as últimas décadas tem aumentado os recursos destinados à saúde de forma constante. No ano fiscal de 2002-2003, o orçamento do NHS chegou a quase 10\% do PIB como resultado de uma estratégia de longo prazo estabelecida pelo governo trabalhista de Tony Blair. Ao mesmo tempo em que se produzia esse aumento constante de recursos, houve um profundo processo de transformação na estrutura e na lógica do sistema. Ainda para esse autor, esse processo de transformação implicou a passagem de um sistema tecnocrático e paternalista, sem critérios adequados, a um sistema de distribuição de recursos para um serviço orientado ao consumidor (consumer-oriented service). Para ele, as reformas introduzidas foram importantes em dois aspectos. Em primeiro lugar por causa de uma política de longo prazo de aumento de gastos em saúde; em segundo, pelo processo de redesenho institucional, que buscava combinar cobertura universal e compreensiva com flexibilidade e capacidade de resposta diante das demandas e necessidades da população 4.

Dopson 5, por sua vez, afirma que as transformações realizadas no NHS devem ser analisadas a partir das mudanças produzidas em um conjunto de esferas: hierarquias, mercados e redes. Para a autora, um elemento fundamental é entender as modificações na gestão do NHS a partir da introdução de um conjunto de práticas provenientes da nova gestão pública (New Public Management - NPM). Essas práticas partiam do pressuposto da necessidade de aumentar a eficiência do sistema de saúde mediante: aumento da concorrência na provisão dos serviços; uso mais eficiente de recursos; criação de medidas de controle de desempenho; e introdução de estilos de gestão do setor privado. No entanto, essa visão gerencialista tem sido criticada por introduzir uma ideologia baseada no mercado, invadindo com suas práticas setores do serviço público orientados por outra lógica de gestão 5 .

O papel do Novo Trabalhismo no processo de reestruturação do NHS é destacado no trabalho de Mohan 6, para quem as reformas realizadas pelo governo de Tony Blair aprofundavam a lógica estabelecida pelo governo conservador, aumentando o papel dos interesses privados na provisão de serviços de saúde. O processo de privatização e mercantilização tem ampliado a importância do setor privado na área da saúde e modificado a relação entre Estado, NHS e setor privado, com importantes consequências para o futuro da saúde no país 6 .

Talbot-Smith \& Pollock 7 também destacam o processo de transição pelo qual tem passado o NHS. Para os autores, o NHS, "um sistema público de assistência em saúde prestada e fornecida pelo Estado, está sendo substituído por um mercado de assistência em saúde, no qual os provedores públicos de serviços competem com os privados pelos fundos do NHS, com contratos legais e regulamentações externas substituindo a responsabilidade política direta" 7 (p. 1). Essa transição estaria sendo muito rápida e teria uma abrangência bastante maior do que os especialistas têm destacado. Nesse processo de mudança, as antigas formas de gestão, organização e provisão da saúde pública foram substituídas por novas estruturas, agências e lógicas 7 .

O objetivo do presente texto é descrever a trajetória de reformas no sistema de saúde britânico, que pode ser considerado como paradigmático no desenvolvimento de grandes tendências na área, particularmente por ser uma referência internacional fundamental no assunto. $\mathrm{O}$ artigo busca apresentar especificamente o processo de reformas realizadas a partir da década de 1990, no NHS, e suas consequências do ponto de vista da reestruturação do sistema de saúde. Para realizar esse objetivo será utilizada um bibliografia secundária que discute as reformas, assim como alguns dos documentos oficiais mais importantes que fundamentaram o processo.

\section{A reforma do governo conservador: eficiência e capacidade de resposta}

Com a crise econômica dos anos 1970 e o surgimento do neoliberalismo, especificamente no Reino Unido, começaram fortes questionamentos sobre a sustentabilidade financeira do Estado de bem-estar social construído no período do pós-guerra. Nesse período, também começaram a aparecer argumentos sobre os supostos altos custos do NHS e o Partido Conservador passou a defender a reforma do sistema de saúde para, conforme seu argumento, melhorar a sua eficiência econômica.

Do ponto de vista ideológico, o governo conservador acreditava que o NHS e outros serviços públicos deveriam utilizar as práticas de gestão do setor privado. Em outubro de 1983, foi publicado 
o Relatório Griffiths, apresentado como uma carta do Secretário do Estado com um conjunto de recomendações que deviam ser tomadas em relação ao NHS. Como afirma Dopson 5, o Relatório Griffiths pode ser considerado um exemplo da forma como a escola do NPM foi introduzida nas propostas de reforma da administração pública no Reino Unido. O Relatório destaca cinco críticas fundamentais com relação ao NHS: a falta de uma direção estratégica central; a falta de responsabilidade gerencial individual; o fracasso em usar objetivos como guia para a ação gerencial; a negligência com relação ao desempenho; a negligência com relação ao consumidor 5.

O Relatório apontava a necessidade de introduzir uma nova forma gestão em que se atribuía a uma pessoa, nos diferentes níveis da organização, a responsabilidade de planejar, implementar e controlar o desempenho, abandonando o modelo prévio de construção de consensos formais sobre o processo de decisão - que tinha sido característico na gestão do NHS 5.

No entanto, foi somente no começo de 1990 que o governo anunciou uma proposta de reforma mais ambiciosa do NHS, com base no diagnóstico apresentado no White Paper Working for Patients, e que avançava sobre as questões colocadas pelo Relatório Griffiths. No novo documento, o governo conservador apresentava o que seriam os principais problemas do NHS: problemas de financiamento; baixa qualidade do atendimento; tempo de espera prolongado para os tratamentos não emergenciais; e diferenças nos padrões de atendimento nas distintas regiões. O governo de Margaret Thatcher procurava introduzir novas estratégias gerenciais para superar os problemas colocados pelo diagnóstico e tornar o sistema mais eficiente em termos de relação custo-benefício 5,8 .

A proposta de reforma do NHS (The NHS and Community Care Act) foi aprovada em 1990 e começou a ser implantada no ano seguinte. As medidas buscavam criar um mercado interno dentro do sistema de saúde, separando os compradores e os prestadores de serviços, e incorporando mecanismos de concorrência que, na leitura governamental, melhorariam a eficiência do sistema. Como afirmam Melo et al. 8, isso implicou importantes mudanças na estrutura interna do NHS com relação ao papel dos diferentes atores no sistema, aos mecanismos de financiamento e gestão, e à integração entre níveis de atenção.

Antes da reforma, os hospitais, centros de saúde e outros serviços médicos eram administrados diretamente pelas autoridades de saúde locais, que atuavam como agentes do Ministério da Saúde (Department of Health). Como afirmam Melo et al. 8, o NHS era composto por 14 autoridades sanitárias regionais (regional health authorities - RHA) e também existiam, no País de Gales, na Escócia e na Irlanda do Norte, escritórios regionais do NHS que supervisionavam estas autoridades (district health authorities - DHA) responsáveis pelo planejamento e operação de hospitais e unidades de saúde. A rede hospitalar era fundamentalmente pública, com $80 \%$ dos leitos pertencentes a hospitais públicos. Do ponto de vista do financiamento dos serviços de saúde, as autoridades distritais de saúde recebiam financiamento público com base no histórico de atendimentos realizados e repassavam estes recursos para hospitais e unidades de saúde.

De acordo com a proposta, afirmam Tanaka \& Oliveira 9, os provedores competiriam oferecendo serviços de saúde com determinada qualidade e custos como forma de atrair os compradores interessados. Os compradores, por sua vez, teriam liberdade para escolher os diversos provedores e poderiam adquirir serviços fora do seu distrito. As DHA continuariam sendo responsáveis pelo planejamento das ações de saúde com base nas necessidades da população. Mas, com a reforma, essas autoridades receberiam recursos públicos, de acordo com o perfil da população do distrito, para contratar os serviços de saúde. Os outros compradores de serviços de saúde passariam a ser grupos de atenção primária denominados GP fundholders, formados por grupos de médicos generalistas (general practitioners - GP) com um número determinado de pacientes que receberiam recursos públicos para a contratação de serviços de saúde secundários. Esses serviços contratados pelos GP fundholders poderiam ser de diversa complexidade, dos mais simples aos mais complexos do sistema 8,9 .

A reforma implantada pelo governo britânico possibilitou aos médicos generalistas, que funcionavam desde o início do NHS como porta de entrada do sistema, se organizarem em GP fundholders para o atendimento da população. Para receberem recursos públicos para custeio, compra de medicamentos e investimento, precisavam cobrir as necessidades sanitárias de uma população de 11 mil pessoas. Os médicos generalistas que não fossem parte de nenhum grupo podiam continuar sendo pagos pelas DHA, mas não teriam acesso aos recursos para serviços secundários 9 . 
Outro elemento fundamental da reforma do NHS foi a reorganização da grande maioria dos hospitais públicos em fundações autônomas e independentes chamadas trusts. Essas fundações (trusts) foram organizadas do ponto de vista gerencial como entidades autônomas, embora o diretor executivo fosse indicado pelas autoridades sanitárias nacionais. As novas fundações (trusts) já não recebiam o seu orçamento diretamente das DHA, e o financiamento era realizado mediante um processo de contratualização com as autoridades de saúde, os GP fundholders e o setor privado 8,9 .

Como resultado da reforma, foi criado um mercado interno no sistema de saúde no qual os trusts passaram a concorrer entre si pelos contratos dos GP fundholders e das DHA. No entanto, como sustentam Tanaka \& Oliveira ${ }^{9}$, continuava a existir também uma regulação estatal importante, uma vez que os contratos permitiam que as autoridades controlassem a qualidade dos serviços prestados pelos hospitais, e havia a possibilidade de suspensão caso não fossem cumpridos os padrões de qualidade requeridos.

$\mathrm{Na}$ avaliação de Klein ${ }^{4}$, os trusts se tornaram empresas semi-independentes que deveriam gerenciar seus orçamentos de maneira "responsável” mediante decisões administrativas, mas também criar incentivos de negócios para serem "atrativos" para os contratantes destes serviços. Essa reforma teve impacto também sobre a atuação dos médicos generalistas, que podiam se responsabilizar pela contratação de cuidados adicionais ou deixar esta contratação nas mãos das autoridades de saúde.

Para Klein 4, o objetivo da reforma era claro: a noção que conduziu essas mudanças foi que a concorrência entre provedores para garantir contratos, sejam as autoridades de saúde, sejam os médicos generalistas que optaram por ser parte dos grupos de atenção primária, melhoraria a eficiência e capacidade de resposta.

Para Dopson 5, a reforma conservadora do NHS partia de uma visão gerencialista inspirada no NPM que sustentava a ideia de uma cultura orientada pelo consumidor e pelo desempenho, e que acreditava nas possibilidades colocadas pelos mecanismos de concorrência de mercado como forma de melhorar a eficiência do sistema. Outra questão importante da reforma foi a incorporação da figura do gerente e do diretor administrativo nas estruturas decisórias do NHS, e que deveriam ser o núcleo da nova dinâmica de gestão.

Além da perspectiva gerencialista colocada pela reforma, outro alvo de crítica era o aumento na fragmentação do sistema de saúde. Com a reforma, afirma Pollitt 10, foi criado um grande número de compradores de serviços de saúde, levando a uma fragmentação do sistema e colocando em questão a coerência do conjunto.

Devido a um complexo conjunto de razões, essa reforma implantada pelo governo conservador nunca funcionou da forma como tinha sido planejada originalmente. No entanto, alguns de seus pilares, como a ideia de aumentar a eficiência e a capacidade de resposta, permaneceram como princípios incorporados na tentativa de reforma que seria implantada nos anos seguintes, mas por um governo de signo distinto do conservador.

\section{Um novo NHS: moderno e confiável. A reforma de 1997}

A eleição do governo trabalhista de Tony Blair em 1997, depois de 18 anos de governos conservadores, parecia oferecer ao NHS tempos menos agitados. No entanto, o novo governo também propunha uma modernização no sistema de saúde britânico 11. Para Mohan 6, uma vez no poder o governo trabalhista abraçou diversas ideias pró-mercado e princípios do NPM que serviram de orientação para as propostas de reforma do NHS.

Em dezembro desse ano (1997), o novo governo enviou ao Parlamento uma proposta de reforma - The New NHS: Modern. Dependable - que buscava criar um novo NHS, "moderno e confiável". Nessa proposta afirmava-se que o objetivo do governo era dar às pessoas o melhor sistema de saúde do mundo. No diagnóstico do governo, o sistema demorava tempo demais para oferecer tratamento aos pacientes e a qualidade era variável, sendo então necessária uma série de reformas para melhorar a qualidade e diminuir o tempo de espera para o atendimento dos pacientes.

Na nova proposta dizia-se: “...o mercado interno será substituído por um sistema que chamamos de "atendimento integrado", baseado na parceria e impulsionado pelo desempenho. Ele forma a base para um 
programa de dez anos para renovar e melhorar o NHS por meio de mudanças evolutivas, e não de disfunção organizacional" 12 (p. 7).

Como afirma Ham 11, o documento indicava que o governo de Blair buscava construir um caminho alternativo entre as políticas conservadoras de reforma do NHS e a visão trabalhista tradicional. A construção dessa nova perspectiva em relação à saúde, mas também em relação aos serviços públicos em geral, se relaciona com a construção de um Novo Trabalhismo por parte do governo de Blair.

A proposta, considerada em conjunto, implicava uma série de mudanças profundas no funcionamento do NHS. Ainda que o horizonte de implementação das transformações fosse de dez anos, o ritmo acelerado de mudanças que havia iniciado no governo anterior continuou durante o governo trabalhista. Como afirma Ham 11, o estabelecimento de grupos de atenção primária, agora denominados primary care trusts, foi a inovação mais importante realizada pelo governo e assinalava um movimento de continuidade, com alguns dos elementos fundamentais da reforma realizada pelo governo conservador. Com isso, na avaliação de Ham 11, buscava-se alinhar a responsabilidade clínica e financeira, retomando a experiência de financiamento do governo conservador e estendendo-a para toda a atenção primária.

Para Klein 4,13, a proposta de reforma do governo trabalhista tinha três características principais.

Em primeiro lugar, a nova proposta criticava a anterior pela criação de um mercado interno. Ela colocava a ênfase na cooperação em lugar da concorrência. Entretanto, na avaliação do autor 4, tal diferença era mais retórica que real e a estruturação do sistema em compradores de assistência médica e provedores, como hospitais e empresas de saúde, foi mantida. A principal diferença foi a mudança para contratos de mais longo prazo, passando de contratos de um ano para contratos de três anos.

Uma segunda característica, como apontado anteriormente, foi a criação dos primary care trusts. Diferentemente da reforma do governo conservador, agora a participação nesses grupos era obrigatória. Todos os médicos generalistas, dentistas, farmacêuticos e outros profissionais da saúde primária precisavam se registrar em uma determinada região geográfica. Esses grupos tinham seus próprios orçamentos e eram responsáveis pelo cuidado médico das suas populações. Os primary care trusts passaram a controlar $75 \%$ dos gastos do sistema de saúde $4,7,13$.

Outra característica da proposta foi a ênfase em oferecer serviços da mesma qualidade em todo o país, resgatando a ideia original do NHS. No diagnóstico do governo existiam importantes disparidades regionais no funcionamento do NHS, e suas medidas buscavam homogeneizar o funcionamento do serviço de saúde no país inteiro e monitorar a sua qualidade 4 .

$\mathrm{Na}$ avaliação de Klein 4, inerente a esse programa existia uma tensão. Por um lado, a ênfase na criação de poderosos primary care trusts, ou colocar os médicos generalistas na condução, como o governo disse, sugeria um serviço desdobrado - poder para a periferia, com decisões sobre quais serviços fornecer e como configurá-los deixados em nível local. Por outro lado, a existência nas normas nacionais sugeria um serviço centralizado, com decisões sobre o nível e a configuração dos serviços realizados no centro. Além disso, o programa deixou um vácuo político. Uma vez que a ideia de confiar na disciplina do mercado foi abandonada, o que restou foi a questão do tipo de incentivos que poderiam ser concebidos para recompensar a eficiência ou conformidade com a política governamental. A história do NHS desde 1997 é, de fato, um registro de tentativas de resolver essa tensão e preencher o vácuo político.

Essa tensão era refletida numa estrutura que buscava fortalecer a definição das necessidades desde baixo, enfatizando as prioridades locais em matéria de saúde. Entretanto, ao mesmo tempo, o governo trabalhista anunciou um conjunto de medidas para fortalecer o poder do governo central. Essas medidas buscavam estabelecer um padrão nacional no modelo de atendimento para homogeneizar a atenção em nível nacional, um dos objetivos iniciais da criação do NHS. Um dos problemas que essa reforma buscava superar era a disparidade no escopo, eficiência e qualidade do serviço de atenção entre as diferentes regiões 13 .

Do ponto de vista da atenção primária, outra inovação importante do governo foi a implantação dos NHS Walk-in Centres. Em janeiro do ano 2000, começaram a funcionar quarenta desses centros em trinta cidades da Inglaterra. O objetivo dos NHS Walk-in Centres era melhorar o acesso à saúde de qualidade de forma eficiente e apoiando outros provedores locais do NHS. A implantação dos centros buscava melhorar o acesso por meio de horários amplos, locais diversos e espera mínima para o atendimento. Outro elemento que se buscava era a utilização de software para a avaliação clínica, 
fornecendo cuidados de alta qualidade, reduzindo a demanda de outros serviços de saúde e, portanto, maximizando a eficiência. Na avaliação de Salisbury et al. 14, essa inciativa, junto com outras propostas de atenção primária, como o NHS Direct, serviço telefônico que oferecia informações de saúde 24 horas ao dia, eram parte importante das iniciativas do governo tendentes a modernizar o NHS.

O governo trabalhista também buscou desenvolver instrumentos para monitorar e melhorar a qualidade dos serviços de saúde no país. Como afirma Walshe 15, foi criada a Comissão de Melhoria da Saúde (Commission for Health Improvement), vinculada diretamente ao Secretário de Estado em Saúde, encarregada de monitorar, assegurar e melhorar a qualidade da saúde no país. As principais funções da comissão incluíam liderança na governança clínica, análise dos arranjos locais de governança via revisão de provedores do NHS, revisão e implementação das orientações nacionais do NHS, supervisão de incidentes externos e análise de problemas graves e persistentes na atenção à saúde. Como sustenta o mesmo autor, embora outras formas de inspeção tenham sido usadas em partes do NHS britânico no passado, essa era "a primeira vez que uma agência com uma competência tão ampla para melhoria da qualidade foi criada” 15 (p. 191).

O período posterior à reforma de 1997 foi um tempo em que se tentou resolver a tensão anteriormente descrita entre a tentativa de ter uma política nacional de saúde, com diretrizes centralizadas, e o processo de descentralização das decisões com a implantação dos primary care trusts. Vinculado a isso, existia certo vácuo político, afirma Klein 4, como resultado da implementação da reforma. A reforma tinha deixado de lado a ideia de um mercado interno, proposta pelo governo conservador, no entanto, não tinha colocado outros tipos de incentivos para melhorar a eficiência da atenção ou para se alinhar com diretrizes políticas formuladas pelo governo central.

Klein 4 sustenta que no primeiro período do governo trabalhista essa tensão foi resolvida por meio de um processo de centralização das decisões. Em sua origem, o NHS tinha sido desenhado para ser um "modelo de comando e controle" na forma de organização do cuidado em saúde. No entanto, do ponto de vista da sua implantação efetiva, nunca tinha funcionado dessa forma. Para Klein, o NHS podia ser descrito de forma mais adequada como um modelo de "exortação e esperança”, no qual o governo central exortava a adoção das medidas em nível local e tinha a esperança de que isto fosse feito. Com base nas medidas implantadas pelo governo trabalhista a estrutura organizacional do NHS foi redesenhada e foram estabelecidas de forma mais clara as competências dos diferentes níveis do sistema. A criação da Comissão de Melhoria da Saúde e do Instituto Nacional de Excelência em Saúde e Assistência (National Institute for Clinical Excellence) exerceram um papel fundamental para a construção de um modelo mais centralizado de gestão e com o estabelecimento de diretrizes de saúde nacionais 4 .

Essa forma de gestão possibilitou que o NHS funcionasse como um sistema mais centralizado, permitindo um comando e controle do governo central. Ainda que historicamente a estrutura do NHS implicasse algum grau de centralização por causa do mecanismo de financiamento via impostos nacionais, tinha existido até esse período um grau importante de autonomia em seu funcionamento. Uma das consequências da reforma realizada pelo governo trabalhista foi um enorme aumento no grau de interferência do governo central. Essa centralização deu lugar a críticas e também a uma avaliação do próprio governo que serviu de fundamento para as mudanças que seriam realizadas nos anos seguintes 4 .

Embora esteja fora dos objetivos deste artigo discutir a criação de NHS regionais no País de Gales, na Escócia e na Irlanda do Norte, como parte da devolução parcial de direitos, é importante mencionar que a eleição do governo Blair teve papel importante neste processo. Como afirma Ham 11, a organização do NHS nesses países sempre apresentou algumas particularidades com relação à Inglaterra. No entanto, a partir de 1999, com a criação de um parlamento escocês e de uma assembleia galesa com poderes sobre serviços como o NHS, houve uma crescente divergência na forma de estruturação da saúde nas partes constituintes do Reino Unido. A criação de NHS regionais recolocava a questão, assinalada anteriormente, da centralização/descentralização das políticas de saúde e reabria um debate que tinha sido resolvido no momento da criação do NHS quando prevaleceu a posição de Aneurin Bevan, que defendia que o NHS devia ser estabelecido como um serviço nacional, contra a opinião de Herbert Morrison, que preferia o controle local do serviço 11,16,17. 


\section{O NHS e a nova ênfase no localismo}

Depois de cinco anos no poder, o governo de Blair começou a avaliar que as propostas centralizadoras com relação ao NHS não tinham sido totalmente bem-sucedidas, e ganhou força a ideia de devolver a responsabilidade pelo funcionamento do NHS para o nível local. Essa ideia sobre a descentralização como forma de resolver os problemas de gestão dos serviços públicos começou a ser discutida internamente dentro do Novo Trabalhismo no início dos anos 200018.

No ano de 2002, o governo delineou uma nova estratégia com relação à gestão do NHS, para tentar superar alguns dos impasses colocados pela reforma de 1997. Essa nova estratégia, chamada pelas autoridades de "localismo real", buscava garantir a provisão de saúde pública coletivamente, de forma moderna, justa e orientada pelo consumidor 18 .

No discurso intitulado Localism: From Rhetoric to Reality, pronunciado em 2003, o Secretário de Estado de Saúde, Alan Milburn, afirmou: "Acredito que podemos ganhar o debate a favor de investimentos e a reforma do serviço público, mas para isto temos de aceitar que a era dos serviços públicos de tamanho único está acabada e que a abordagem da centro-esquerda hoje deve se basear em descentralização, diversidade e escolha" 18 .

O governo alegava que a nova proposta de reforma era a melhor estratégia para garantir os valores do NHS, entretanto, era necessário mudar a estrutura de funcionamento do sistema. Os fins do NHS permaneceriam os mesmos, um sistema universal que garantisse saúde para todos, independentemente da possibilidade de pagar por ela. O que era necessário mudar era a forma de alcançar esses fins, pois já não era possível uma forma de gestão baseada na ideia de monopólio público.

$\mathrm{Na}$ avaliação do governo, o NHS apresentava enormes pontos positivos, mas também tinha fraquezas que precisavam ser corrigidas. Um dos pontos a ser corrigido estava relacionado com a distância construída entre a gestão central do NHS e as necessidades locais de provisão de saúde. O Reino Unido, sustentava o documento, não era um país uniforme, existiam grandes diferenças regionais que precisavam ser levadas em conta na formulação de políticas públicas. O NHS precisava ser organizado de forma que desse atenção às necessidades de cada localidade, confiando na capacidade de pessoas de inovar e de tomar iniciativas. O documento afirmava, ainda, que as decisões sobre a gestão da saúde deviam ser tomadas nos hospitais e centros de saúde e não de forma centralizada pelo governo nacional. O processo de descentralização das decisões seria a estratégia adotada pelo governo para melhorar a eficiência da prestação dos serviços de saúde 19.

A nova estratégia do governo indicava o reconhecimento de que um modelo centralizado de controle e comando, como o que fora proposto na reforma de 1997, tinha efeitos perversos. Mas também era uma estratégia para tirar a responsabilidade dos problemas do NHS do governo central e dividir a responsabilidade com os governos locais 4.

Como afirma Allen 17, do ponto de vista da provisão de serviços de saúde, a proposta de descentralização passava pela introdução das Foundation Trusts. A criação dessas organizações buscava atender a elementos importantes para o governo, uma melhoria da eficiência e a orientação pelo consumidor. Um elemento adicional, continua o autor, dessas inovações era a criação de um novo sistema financeiro, que seria implantado a partir de abril de 2005, baseado no pagamento por resultados.

Como afirmam Allen et al. 19, a implantação das Foundation Trusts buscava outorgar maior autonomia em relação ao governo central e garantir o envolvimento de funcionários, pacientes e servidores públicos na gestão dos hospitais. O objetivo de aumentar a autonomia do poder central se articulava com a introdução de mecanismos de mercado que tinham sido incorporados anteriormente na estrutura do NHS. Com esse processo de descentralização e de mudança na governança, o governo pretendia aumentar a capacidade de resposta do sistema de saúde às demandas das comunidades locais e torná-las mais receptivas aos desejos dos consumidores 19.

Para Klein 4, a nova proposta de reforma buscava introduzir unidades organizacionais semiautônomas (Foundation Trusts) com capacidade para decidir sobre estruturas de salários para médicos e outros profissionais, e com autonomia para captar recursos do mercado para financiar os serviços de saúde. Havia diferenças importantes entre as organizações de saúde existentes até aquele momento e as novas que seriam criadas a partir da reforma anunciada. As organizações de saúde eram supervisionadas pela Secretaria da Saúde, e na nova proposta a responsabilidade pela fiscalização das NHS Foundation Trusts passava a ser de um ente regulatório independente. No primeiro momento foram 
selecionados os trinta grupos mais bem avaliados pelo governo e se permitiu que eles se transformassem nas novas NHS Foundation Trusts 20.

A introdução das NHS Foundation Trusts implicava uma mudança muito importante de orientação, no sentido de estabelecer um sistema menos centralizado e mais orientado pelo consumidor. Dessa forma, também vemos uma mudança importante na ênfase colocada pelo governo trabalhista - da retórica da reforma anterior, que destacava a importância de colocar os agentes de saúde na gestão do sistema, passou-se a uma retórica que ressaltava a escolha do paciente. O objetivo do governo era orientado pelo princípio de dar aos pacientes o direito de escolha, permitindo a eleição de especialistas e tratamentos, assim como das cirurgias eletivas 20.

A criação das NHS Foundation Trusts pode ser considerada uma das transformações mais importantes na história do NHS e implicava uma nova forma de provisão e gerenciamento do sistema de saúde britânico. A mudança levava a uma reconfiguração do ponto de vista da provisão do serviço, que continuava sendo público, no entanto, não diretamente ofertado pelo Estado. Ainda que as NHS Foundation Trusts continuassem a fazer parte do NHS, passavam a ser definidas, de acordo com a saúde e assistência social (Health and Social Care - Community Health and Standards Act, 2003), como organizações independentes denominadas corporação de utilidade pública (public benefit corporations), com o objetivo de fornecer serviços em saúde para pacientes e usuários do NHS de acordo com os princípios e padrões estabelecidos pelo próprio NHS 4.

Essas organizações passaram a ter maior liberdade e autonomia para fazer a gestão dos recursos e realizar investimentos. A nova estrutura deveria ter uma relação estreita com as comunidades locais nas quais prestava serviços, e os membros destas comunidades tinham a possibilidade de participar na gestão dessas organizações. O objetivo do governo era que as comunidades locais tivessem papel mais ativo na definição das necessidades de prestação de serviços em saúde nas suas localidades. Dessa forma, as NHS Foundation Trusts poderiam, em contrapartida, ofertar serviços mais próximos às necessidades das comunidades locais e, agora, com liberdade para desenvolver estratégia de oferta de serviços em saúde de acordo com as expectativas da população 18.

Essas mudanças foram acompanhas por alterações na estrutura interna de financiamento do sistema. O sistema que funcionava, até a reforma, com base na ideia de "pagamentos por resultados", foi gradualmente alterado para outro sistema com o objetivo, nas palavras do próprio documento do governo, de "apoiar a escolha do paciente, garantindo que diversos provedores possam ser financiados de acordo com a escolha do paciente a ser tratado" 4 (p. 940). O documento publicado pelo governo britânico em 2002, intitulado Reforming NHS: Financial Flows. Introducing Payment by Results, descrevia essa estratégia do governo com relação ao financiamento do NHS 20.

Com esse conjunto de medidas colocadas em prática pelo governo trabalhista, somadas às anteriores já analisadas, produz-se a maior reorganização na forma de provisão e gestão da saúde no Reino Unido nos últimos cinquenta anos, conforme Figura 1.

\section{Considerações finais}

Como exposto no início do texto, a reforma do NHS britânico dos últimos anos apresenta elementos interessantes para pensar os problemas, desafios e tensões inerentes às transformações dos sistemas de saúde pública das sociedades contemporâneas. Esse processo de reforma, o mais importante pelo qual passou o NHS desde a sua criação, implicou três momentos diferentes.

Em primeiro lugar, a reforma do governo conservador realizada em 1991, que envolveu uma mudança fundamental na trajetória do NHS, colocando uma nova visão gerencial na gestão do sistema, com o objetivo de melhorar sua eficiência. Para alcançar tal fim a reforma se propunha a introduzir mecanismos de mercado, com a criação de um mercado interno, separando compradores e prestadores de serviços como forma de melhorar a eficiência do sistema.

Um segundo momento do processo de reforma foi o das mudanças implantadas pelo governo trabalhista de Tony Blair, em 1997. As novas medidas buscavam colocar uma ênfase maior na cooperação do que na concorrência, no entanto, esta nova ênfase parecia ser mais uma questão retórica do que uma mudança fundamental com relação às medidas anteriores. Um elemento adicional das medidas 


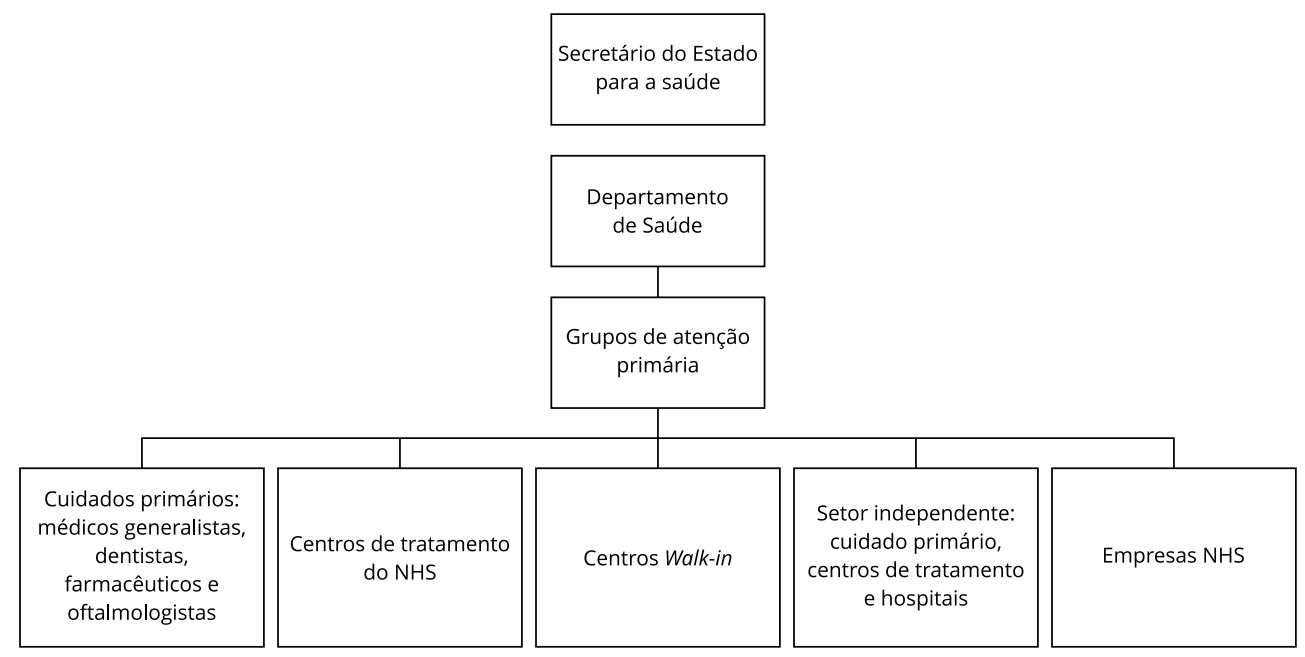

Fonte: Talbot-Smith \& Pollock 7 .

implantadas em 1997 foi a tentativa de criar um padrão nacional para avaliar o funcionamento do sistema de saúde.

O último momento da reforma foi elaborado com base em uma estratégia denominada "localismo real", que estabelecia que a provisão de serviços de saúde deveria ser orientada pelo consumidor, ser menos centralizada e ter maior protagonismo das autoridades locais. Com as mudanças propostas buscava-se dar aos consumidores a possibilidade de eleição de especialistas e tratamentos.

Esse processo de reforma implicou uma mudança no sentido de introduzir mecanismos de mercado no interior do sistema de saúde, tanto pelas modificações propostas pelo governo conservador quanto por aquelas propostas pelo governo trabalhista. O pressuposto era que os mecanismos de mercado melhorariam a eficiência na distribuição dos recursos. Na estratégia do localismo real também estava implícita a ideia da orientação pelo consumidor como forma de melhorar a oferta de serviços, claramente uma ideia de conotação neoliberal.

As reformas tiveram como consequência uma transformação profunda do NHS e do papel do Estado como provedor e regulador dos serviços de saúde no Reino Unido. Como afirmam Talbot-Smith \& Pollock 7, o modelo que gradualmente emergiu como consequência desse processo foi o de um NHS contratando diversos serviços de saúde entre vários prestadores, tanto públicos como privados. O NHS passou a ser cada vez menos um provedor direto de serviços de saúde para a população e cada vez mais um contratante financiado pelo Estado. Por outra parte, o antigo sistema de responsabilização política baseada no ethos do serviço público mudou para um modelo com base em mecanismos de direito privado 7 .

Ao analisar a reforma realizada no NHS, para além de trazer elementos que permitam compreender o debate atual proporcionado pelas iniciativas lideradas por Boris Johnson que visam a empreender nova reforma no NHS a partir de 2021, é possível encontrar convergências com outras propostas de reforma de sistemas públicos de saúde, que se inspiraram em sua conformação no modelo britânico, por exemplo no caso do Brasil. Em um artigo publicado recentemente sobre a reforma do Sistema Único de Saúde, colocava-se a necessidade de uma integração entre o setor público, responsável pelo aporte de dinheiro, e o setor privado responsável "pela gestão 'eficiente' e 'moderna' dos recursos" 21 . As semelhanças não parecem pura coincidência. Todavia, esse não era o escopo do presente artigo, que procura levantar alguns elementos para pensar se as reformas implantadas no NHS também podem ser vislumbradas em outros países que têm sistemas universais de saúde. 


\section{Colaboradores}

Todos os autores participaram igualmente da produção do artigo.

\section{Referências}

1. Immergut E. Health politics: interests and institutions in Western Europe. Cambridge: Cambridge University Press; 1992.

2. Almeida C. Reformas de sistemas de saúde: tendências internacionais, modelos e resultados. In: Giovanella L, Escorel S, Lobato, LVC, Noronha JC, Carvalho AI, organizadores. Políticas e sistemas de saúde no Brasil. Rio de Janeiro: Editora Fiocruz; 2014. p. 759-801.

3. Escoval A, Ribeiro RS, Matos TT. A contractualização em cuidados de saúde primários: o contexto internacional. Rev Port Saúde Pública 2010; (9):41-57.

4. Klein R. Britain's National Health Service revisited. N Engl J Med 2004; 350:937-42.

5. Dopson S. Changing forms of managerialism in the NHS: hierarchies, markets and networks. In: Gabe J, Calnan M, editors. The new sociology of the health service. London/New York: Routledge; 2009. p. 37-9.

6. Mohan J. Visions of privatization: new labour and the reconstruction of the NHS. In: Gabe J, Calnan M, editors. The new sociology of the health service. London/New York: Routledge; 2009. p. 79-80.

7. Talbot-Smith A, Pollock AM. The new NHS. A guide. London/New York: Routledge; 2006.

8. Melo MA, Costa NR, Silva PLB. Inovações organizacionais em política social: o caso da Grã -Bretanha. Revista do Serviço Público 1999; 50:5-31.

9. Tanaka OY, Oliveira V. Reforma(s) e estruturação do Sistema de Saúde Britânico: lições para o SUS. Saúde Soc 2007; 16:7-17.

10. Pollitt C. Inglaterra. In: Ramos S, Vinocur P, editores. Sistemas de salud y reformas: diferentes respuestas a la relación entre Estado y el mercado. Buenos Aires: Centro de Estudios de Estado y Sociedad; 2000. p. 41-79.

11. Ham C. Health policy in Britain. London: Macmillan; 1999.

12. Department of Health. The new NHS: modern, dependable. https://www.gov.uk/govern ment/uploads/system/uploads/attachment_ data/file/266003/newnhs.pdf (acessado em 20/Jul/2020).

13. Klein R. What's happening to Britain's National Health Service? N Engl J Med 2001; 345:305-8

\section{Informações adicionais}

ORCID: Javier Amadeo (0000-0002-3941-2163);

Rosemarie Andreazza (0000-0002-3332-2183);

Ademar Arthur Chioro dos Reis (0000-0001-7184-

2342).

14. Salisbury C, Chalder M, Scott TM, Pope C, Moore L. What is the role of walk-in centres in the NHS? BMJ 2002; 324:399-402.

15. Walshe K. Improvement through inspection? The development of the new Commission for Health Improvement in England and Wales. Qual Health Care 1999; 8:191.

16. Hawkes N. How different are NHS systems across the UK since devolution? BMJ 2013; 346:f3066.

17. Allen P. New localism in the English NHS: what is it for? Health Policy 2006; 79:244-52.

18. Political Speech Archive. Alan Milburn - 2003 speech on localism. http://www.ukpol.co.uk/ alan-milburn-2003-speech-on-localism/ (acessado em 16/Jul/2020).

19. Allen P, Townsend J, Dempster P, Wright J, Hutchings A, Keen J. Organizational form as a mechanism to involve staff, public and users in public services: a study of the Governance of NHS Foundation Trusts. Soc Policy Adm 2012; 46:239-57.

20. Department of Health. A short guide to NHS Foundation Trusts. https://www.wwl.nhs. uk/Library/Foundation_Trust/Foundation_ Trust_Guide.pdf (acessado em 24/Jul/2020).

21. Mathias M, Torres R. A estranha "reforma do SUS” que Rodrigo Maia trama. Outrasaúde 2020; 27 jul. https://outraspalavras.net/outra saude/osetorprivadoeareformadosus/. 


\section{Abstract}

Recent decades have witnessed important transformations in public services that involve changes in the State's role as central agent in the regulation of public-private relations and the definition of levels of public financing. Health system reform proposals are part of the process of social transformations that have affected various nations. However, changes in the linkage between different dimensions are the result of both broad processes of social transformation and specific experiences and timelines, essential for understanding the results of this process. In recent decades, the British National Health Service (NHS) underwent the most important reform process since its creation in 1948. This process began with a set of measures implemented by the Conservative government in 1991, which were continued through alterations introduced in 1997 and 2002. An analysis of the NHS reform not only provides elements for understanding the current debate sparked by the initiatives led by Boris Johnson and aimed at a new NHS reform starting in 2021; such analysis also allows identifying convergences with other reform proposals in public health systems that were shaped under inspiration from the British model, as in the case of Brazil. The current article aims to discuss and analyse a case of health system reform that can be considered paradigmatic for the development of major trends in this field. The article seeks to specifically analyse the reform processes carried out since the 1990s in the NHS and their consequences for the health system's restructuring.

Health System; Health Care Reform; Health Services; Public Health

\section{Resumen}

En las últimas décadas asistimos a importantes transformaciones en los servicios públicos que implican cambios en el papel del Estado, como agente central en el proceso de regulación de las relaciones público-privadas, así como en la definición de los niveles de financiación pública. Las propuestas de reforma de los sistemas de salud forman parte del proceso de transformaciones sociales que afectaron a diferentes países. No obstante, los cambios en la coordinación de las diferentes dimensiones son resultado, tanto de procesos amplios de transformación social, como también de experiencias y factores temporales específicos fundamentales para entender los resultados de ese proceso. El Servicio Nacional de Salud británico (NHS) pasó en las últimas décadas por el proceso de reforma más importante desde su creación en 1948. Este processo comenzó con un conjunto de medidas implementadas por el gobierno conservador en 1991, pero que continuaron con las modificaciones realizadas en 1997 y en 2002. Al analizar la reforma realizada en el NHS, además de aportar elementos que permitan comprender el debate actual, debido a las iniciativas lideradas por Boris Johnson que tienen como objetivo emprender una nueva reforma en el NHS, a partir de 2021, es posible encontrar convergencias con otras propuestas de reforma de sistemas públicos de salud, que se inspiraron en su conformación en el modelo británico, como, por ejemplo, en el caso de Brasil. El objetivo de este trabajo es discutir y analizar un caso de reforma en un sistema de salud que puede ser considerado como paradigmático en el desarrollo de grandes tendencias en el área. El texto busca analizar específicamente el processo de reformas realizadas, a partir de la década de 1990, en el NHS, y sus consecuencias desde el punto de vista de la reestruturación del sistema de salud.

Sistemas de Salud; Reforma de la Atención de Salud; Servicios de Salud; Salud Pública
Recebido em 05/Ago/2020

Versão final reapresentada em 21/Jan/2021

Aprovado em 06/Fev/2021 